\title{
ANALISANDO O COMPORTAMENTO DOS GASTOS PUBLICOS NOS MUNICÍPIOS CEARENSES
}

\author{
José Geraldo Araújo Correia \\ Tribunal de Contas dos Municípios do Estado do Ceará (TCM-CE) \\ Guilherme Irffi Diniz \\ Universidade Federal do Ceará (UFC) \\ Rodolfo Ferreira Ribeiro da Costa \\ Universidade Estadual do Rio Grande do Norte (UERN)
}

\section{ANALISANDO O COMPORTAMENTO DOS GASTOS PÚBLICOS NOS MUNICÍPIOS CEARENSES}

Resumo: $O$ objetivo deste trabalho é testar a hipótese de flypaper na administração pública municipal do estado do Ceará. Para tal, foi construído um painel de dados com informações sobre os gastos, a arrecadação, a população e as transferências constitucionais para os 184 municípios cearenses entre 1999 e 2009. Tais informações foram extraídas junto à Secretaria do Tesouro Nacional e ao Instituto Brasileiro de Geografia e Estatística. A metodologia utilizada remete-se ao modelo de dados em painel com efeito aleatório. Os resultados destacam que a elasticidade gasto-renda supera a elasticidade gasto-transferência e, portanto, as administrações municipais estão isentas da prática descrita pelo efeito flypaper.

Palavras-chave: Flypaper, gasto, transferência, dados em painel.

\section{ANALYZING THE BEHAVIOR OF THE PUBLIC SPENDING IN CEARÁ'S CITIES}

Abstract: The aim of this study was to test the hypothesis flypaper in municipal public administration in the state of Ceará. To this end, was built a panel with information on expenditures, revenues, population and constitutional transfers to the 184 municipalities of Ceará between 1999 and 2009. Such information was extracted together with the National Treasury and the Brazilian Institute of Geography and Statistics. The methodology used is referred to the panel data with random effect model. The results highlight that the elasticity spending exceeds income-elasticity worn-transfer and, therefore, local governments are exempt from the practice described by flypaper effect.

Keywords: Flypaper, expenditure, transfer, panel data. 


\section{INTRODUÇÃO}

Uma questão-chave na teoria do federalismo fiscal é 0 financiamento de governos descentralizados, principalmente, pela limitada base tributária das esferas inferiores de governo. Diante disso, para os municípios os repasses de verbas federais, especialmente aqueles de caráter constitucional, é um fato indispensável para a gestão de bens e serviços que propiciam 0 atendimento das necessidades locais.

A preocupação geral com esta forma de financiamento é que tal interação entre os níveis de governo distorça os pontos fortes da descentralização. Portanto, entender 0 funcionamento da interação fiscal entre governo federal e municipal, bem como entre estadual e municipal é fator preponderante para a concepção de regimes federalistas.

Nesse sentido, destaca-se a discussão sobre o comportamento dos gastos totais mediante as transferências intergovernamentais e a renda local (aferida pelo PIB municipal). A comparação entre os efeitos dos repasses constitucionais e da renda em relação às despesas públicas propiciam a informação necessária para análise do efeito flypaper, fenômeno observado quando uma previsão de aumento nos repasses tende a estimular mais os gastos do que o crescimento do PIB.

A literatura apresenta vários trabalhos que visam identificar a prática dos gestores municipais caracterizada como efeito flypaper. Tanto do ponto de vista teórico quanto empírico, as análises da gestão das despesas são remetidas às versões do modelo do eleitor mediano, diferenciando-se apenas 0 ambiente no qual os individuos estão inseridos entre cenários com informação perfeita e imperfeita, além do modelo burocrata de maximização do orçamento público.

Exemplos de trabalhos com base no modelo do eleitor mediano em ambientes com informação perfeita são Gramlich e Galper (1973), Gramlich (1977), Courant, Gramlich e Rubinfield (1979), Oates (1979), Filimon, Romer e Rosenthal (1982) e Strumpf (1998).
Considerando ambientes de incerteza, tem-se Turnbull (1992), Cossio e Carvalho (2001) e Mattos, Rocha e Arvate (2011).

Oates (1988) destaca que o modelo do eleitor mediano, dada à hipótese de ilusão fiscal, apresenta apenas uma possibilidade para ocorrência do efeito flypaper, tendo a mesma a necessidade de apoio empírico.

A partir desta descrição, pode-se dizer que este trabalho tem como objetivo verificar se a gestão dos municípios cearenses condiz com a prática definida pela literatura como efeito flypaper.

Para realização de tal propósito estimam-se os efeitos das transferências intergovernamentais e do PIB municipal sobre o comportamento do gasto público dos municípios cearenses. Para estimar esses efeitos, consideram-se os modelos de dados em painel, dado que o Estado é composto por 184 municípios e as informações sobre gastos, arrecadação, população, PIB e transferências consideram o interstício entre 1999 e 2009. Os dados foram obtidos junto à Secretaria do Tesouro Nacional (STN) e ao Instituto Brasileiro de Geografia e Estatística (IBGE).

A comparação do efeito das transferências com o observado para renda em relação às realizações orçamentárias das jurisdições cearenses será 0 argumento responsável por qualificar se a gestão pública municipal condiz com a postura expressa pelo efeito flypaper. Ou seja, a interpretação dos resultados basear-se-á na mensuração das elasticidades gasto-renda e gasto-transferência e, caso o valor da primeira seja inferior ao desta última, tem-se a caracterização do efeito flypaper.

Além desta seção introdutória, este trabalho possui mais quatro seções. A que segue apresentará os principais relatos sobre a averiguação do efeito flypaper de acordo com diferentes correntes teóricas. A terceira expõe o procedimento metodológico, bem como definição das variáveis e seus efeitos esperados sobre o gasto público. A quarta, inicialmente, discutirá as estatísticas descritivas e, em seguida, reporta os resultados e as análises das estimações. E, por fim, são acrescidas as considerações finais. 
2 REVISÃO DA LITERATURA: modelos teóricos

Duas teorias concorrentes dominam a discussão sobre efeito flypaper. Análises baseadas na hipótese de ilusão fiscal e no modelo de maximização do orçamento (burocratic model) formam as principais contribuições referentes ao comportamento das despesas em relação às variações nos repasses intergovernamentais e no PIB municipal.

De acordo com Schwallie (1986), no modelo de ilusão fiscal, o efeito flypaper é um resultado da percepção equivocada do eleitor mediano sobre os aspectos de financiamento $e$ as decisões fiscais resultantes de tal equívoco. Nesses modelos o governo de fato produz 0 montante de bens e serviços exigidos pelos eleitores, sendo que a demanda dos mesmos é baseada na ignorância sobre a forma de financiamento e os custos relacionados à produção.

De acordo com o modelo burocrático, o efeito flypaper é o resultado de um comportamento de maximização do orçamento pelo gestor local, denominado por McGuire (1973) de um modelo de políticos gananciosos. O efeito flypaper é possível no modelo burocrático devido ao conhecimento superior dos burocratas sobre os subsídios e do orçamento. Tais informações possibilitam a obtenção de um orçamento superior ao desejado pelo eleitor mediano. 0 burocrata age estrategicamente liberando informações distorcidas e respondendo a cortes no orçamento, reduzindo gastos com os programas populares e inflando todos os pedidos de orçamento.

Além das correntes discutidas acima, existem algumas outras fontes teóricas que tratam as questões ligadas ao efeito flypaper. Hamilton (1986) detaca que a perda de peso morto criada pela tributação do governo local cria custos adicionais para as receitas fiscais, enquanto que repasses intergovernamentais não apresentam custos adicionais. Logo, o financiamento das despesas a partir de recursos provenientes de transferências intergovernamentais não apresentam prejuízos em termos de potencial econômico para a comunidade como observado no financiamento via arrecadação própria. Tal fato justificaria um efeito mais expressivo das transferências, em relação à renda, sobre as realizações orçamentárias das juridições, propiciando um ambiente com condições para a ocorrência do efeito flypaper.

Outra explicação para 0 referido fenômeno é dada por Quigley e Smolensky (1992) que analisam o impacto dos custos envolvidos em operações de captação de recursos pelos governos locais. Dado que as únicas formas de adquirir fundos para 0 financiamento das despesas é por meio de repasses e da arrecadação própria, o governo local deve avaliar os efeitos dos custos relacionados às mudanças nas alíquotas e transferências recebidas. Como num regime federativo a determinação das alíquotas é realizada anualmente, mudanças na renda disponível dos indivíduos, acompanhadas de mudanças nas alíquotas, exigiria reformulação no planejamento orçamentário de forma mais custosa do que aquela proveniente de alterações no volume de repasses. Portanto, flutuações nos recursos provenientes de alterações na renda provocam variações nos gastos inferiores às provocadas pelas transferências.

Adicionando uma restrição no problema do eleitor mediano, fazendo com que o nível de renda do agente representativo não possa situar-se abaixo de certo nível especificado pelo eleitor mediano, King (1984) observa que níveis mais elevados de produção de serviços pelo governo local exigem uma alíquota de imposto mais elevada, assumindo transferências intergovernamentais como fixas. Em algum momento, o aumento das alíquotas estabelecidas pela autoridade local resultaria em cidadãos mais pobres, isto é, ter renda disponível abaixo do nível especificado pelo eleitor mediano, supondo que todos os grupos de renda são obrigados a pagar o imposto local. É então possível para demonstrar o efeito em termos flypaper. Esta formulação é conhecida na literatura como modelo com restrição de baixa renda. 
Bradford e Oates (1971) apresentam mais duas possibilidades de ocorrência do efeito flypaper. Se por um lado, a falta de estrutura institucional nega a suposição de que 0 processo político é altamente sensível às preferências dos indivíduos, seja por causa das restrições colocadas sobre o nível mais baixo do governo pela autoridade superior, ou porque as regras do próprio governo local possam apresentar falhas por negligência e não por projeto, a ação descomprometida com 0 equilíbrio orçamentário apresenta-se com maior probabilidade. Por outro lado, a falta de hábito ou aprendizagem ocorre quando os cidadãos não sabem e não podem prever corretamente as consequências de uma ação proposta coletiva. Com imprecisões no diagnóstico das preferências, então, a prática imprudente da gestão torna-se provável.

Turnbull e Niho (1986) argumentam que o efeito flypaper é devido à capitalização de imposto decorrente do pagamento de bolsasauxílio, do aumento resultante em base tributária local, levando a maiores receitas e despesas. No entanto, pode-se esperar que, nos anos seguintes, os eleitores busquem alíquotas mais baixas se a sua elasticidade demanda manter-se inalterada.

Com base nessa exposição, este trabalho pretende contribuir com a literatura por analisar a existência (ou não) do efeito flypapper dos gastos municipais cearenses.

\section{ESTRATÉGIA EMPÍRICA, FONTE E DESCRIÇÃO DOS DADOS}

Para identificar se as gestões municipais cearenses são condizentes com a postura definida pelo efeito flypaper, estimar-se-ão as elasticidades gastos-transferências e gastosrenda a partir de modelos para dados em painel, na sua versão estática estimado por efeito aleatório e efeito fixo, cuja determinação do efeito predominante se dará a partir do teste de Hausman.

A justificativa para utilização da metodologia de dados em painel baseia-se nas características inerentes à estrutura de organização dos dados, 184 municípios cearenses ao longo dos anos de 1999 a 2009, sendo o referido período justificado por ser 0 único que contemple de forma contínua 0 conjunto de informações municipais. Isto possibilita a redução a níveis de colinearidade relativamente baixos entre as variáveis explicativas, aumento do número de informações a partir da combinação dos dados de cortes com séries de tempo, investigar um conjunto de indivíduos heterogêneos, além de permitir o acompanhamento da dinâmica de ajustamento ao longo do tempo.

A abordagem estática do modelo de dados em painel admite a hipótese de exogenidade estrita, ou seja, que não existe relação entre as variáveis explicativas e 0 termo de erro. A primeira tarefa que se impõe a essa abordagem corresponde à escolha entre os modelos de estimação, com predomínio entre os efeitos fixos e efeitos aleatórios, a depender fundamentalmente do objetivo que se propõe o estudo e do contexto no qual foram coletados os dados ou, ainda, pode-se optar pela realização do teste de Hausman, que compara se existem diferenças entre os parâmetros estimados pelas especificações de efeito fixo ou aleatório, tendo como hipótese nula a indicação do modelo com efeitos aleatórios como o mais adequado para se tratar o problema em questão.

\subsection{Modelo com Efeito Fixo}

A formulação com efeito fixo é idealizada a partir da suposição de que existem caracteríticas que diferenciam os indivíduos e que tal heterogeneidade é captada pelo processo de estimação através do intercepto, que se diferencia de indivíduo para indivíduo, ou seja, supõe-se que o termo constante sofre variações no espaço, mas é constante ao longo do tempo. 0 modelo com efeito fixo pode ser apresentado como segue:

$$
Y_{i t}=\alpha_{i}+\beta X_{i t}+\epsilon_{i t}
$$

onde, a constante $\alpha_{i}$ é diferente para cada indivíduo, captando heteregonidades variantes no espaço, mas invariantes no tempo. Como as estimativas de $\beta$ não variam entre os 
indivíduos e nem ao longo do tempo, a heterogenidade predominante entre os individuos é capturada pelo intercepto.

0 processo de estimação do modelo com efeitos fixos é realizado a partir dos desvios das variáveis em relação aos seus respectivos valores médios. Seja $Q$ um vetor

que projeta as variáveis na forma de desvios, tem-se

$$
\begin{aligned}
& Q Y_{i t}= Q\left(\alpha_{i}+\beta X_{i t}+\epsilon_{i t}\right) \\
& \tilde{Y}_{i t}=\tilde{\alpha}_{i}+\beta \tilde{X}_{i t}+\tilde{\epsilon}_{i t}
\end{aligned}
$$

Sendo $\tilde{\alpha}_{i}=0$, verifica-se

$$
\tilde{Y}_{i t}=\beta \tilde{X}_{i t}+\tilde{\epsilon}_{i t}
$$

onde: $\quad \tilde{Y}_{i t}=Q Y_{i t}, \quad \tilde{X}_{i t}=Q X_{i t}$,

$\tilde{\alpha}_{i}=Q \alpha_{i}$ e $\tilde{\epsilon}_{i t}=Q \epsilon_{i t}$.

Assim, trabalhando com os desvios das variáveis em torno dos seus respectivos valores médios, tem-se a eliminação do termo de heterogeneidade $\tilde{\alpha}_{i}$, já que $Q \alpha_{i}=0$. 0

vetor de estimadores de efeitos fixos, $\hat{\beta}$, pode

ser obtido por mínimos quadrados ordinários:

$$
\hat{\beta}=\left(\tilde{x}^{\prime} \tilde{x}\right)^{-1} \tilde{x} \tilde{y}(5)
$$

Portanto, caso o teste de Hausman indique que o modelo que melhor representa 0 conjunto de dados seja o de efeito fixo, será utilizada a equação (5) para se obter o conjunto de parâmetros que indicaram 0 efeito das transferências e da renda sobre os gastos, de modo que seja avaliada a ocorrência do efeito flypaper.

\subsection{Modelo com Efeito Aleatório}

Apesar de assumir que os individuos possuem características que os distinguem, assim, como o modelo com efeito fixo, a versão do modelo para dados em painel com efeito aleatório que 0 termo que capta a heterogenidade entre os agentes é um componente do termo de erro:

$$
\begin{aligned}
& Y_{i t}=\alpha+\beta X_{i t}+w_{i t},(6) \\
& \text { onde } \\
& w_{i t}=\eta_{i}+\epsilon_{i t}(7)
\end{aligned}
$$

sendo, $\eta_{i}$ é 0 efeito aleatório não observado e $w_{i t}$ um componente de erro idiossincrático com as seguintes propriedades:

$$
E\left(w_{i t}\right)=0
$$

$$
\begin{aligned}
& \operatorname{Var}\left(w_{i t}\right)=\sigma_{\eta}^{2}+\sigma_{\varepsilon}^{2} \\
& \operatorname{Cov}\left(w_{i t}, w_{i s}\right)=\sigma_{\eta}^{2}, \forall t \neq s . \\
& \operatorname{Cov}\left(w_{i t}, w_{j t}\right)=0, \forall i \neq j .
\end{aligned}
$$

Os dois primeiros itens destacam que 0 termo de erro possui média zero e variância constante, ou seja, o erro é do tipo homocedástico. A terceira retrata a autocorrelação existente entre os termos de erro de um mesmo indivíduo. Por fim, a quarta propriedade diz que os erros de indivíduos diferentes são não correlacionados para um mesmo período de tempo.

Apesar de se caracterizar como homocedástico, as propriedades do termo de erro mostram que a autocorrelação se faz presente e, portanto, os estimadores de Mínimos Quadrados não são capazes de atender as condições relatadas no teorema de Gauss-Markov, ou seja, dada a relação temporal existente entre os termos de erro, o estimador de MQO não possui a característica de eficiência. A solução para este problema é idealizada a partir do método de Mínimos Quadrados Generalizados, que se baseia na ponderação da matriz de variáveis explicativas de forma a proporcionar um estima robusta para variância do modelo e, assim, dos parâmetros. Seja $\Omega^{-1}=P^{\prime} P$, o estimador de

MQG é obtido a partir de (6):

$$
P Y_{i t}=P\left(\alpha+\beta X_{i t}+w_{i t}\right)
$$




$$
\tilde{Y}_{i t}=\tilde{\alpha}+\tilde{\beta} \tilde{X}_{i t}+\widetilde{w}_{i t}
$$

Aplicando o método de Gauss e reescrevendo em formato matricial, chega-se ao estimador de efeito aleatório:

$$
\begin{array}{r}
\tilde{\beta}=\left(\tilde{X}^{\prime} P^{\prime} P \tilde{X}\right)^{-1} \tilde{X}^{\prime} P^{\prime} P \tilde{Y} \\
\tilde{\beta}=\left(\tilde{X}^{\prime} \Omega^{-1} \tilde{X}\right)^{-1} \tilde{X}^{\prime} \Omega^{-1} \tilde{Y}(8)
\end{array}
$$

Finalmente, caso a heterogeneidade observada para o conjunto de informações utilizado seja tal que o teste de Hausman recomende o modelo com efeito aleatório como 0 indicado para mensurar o comportamento do gasto municipal no Ceará e, assim, verificar se o mesmo condiz com a definição de flypaper, poder-se-á utilizar a equação (8) para obter-se o vetor de parâmetros que balizará a referida avaliação.

\subsection{Estratégia Empírica, Fonte, Descrição dos Dados e Análise Descritiva}

O modelo empírico a ser estimado para identificar se os municípios cearenses corroboram com a definição de efeito flypaper, se baseia no modelo do eleitor mediano e tem os gastos como função das transferências, PIB e Impostos, além da contingente populacional, expresso por meio da seguinte equação:

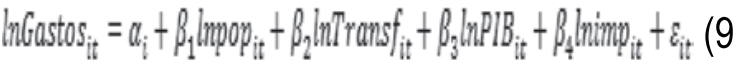

Sendo lngastos $_{i t}$ representa 0 logaritmo das despesas totais do i-ésimo município e no período $t$, Inpop it o logaritmo do número de habitantes residentes no i-ésimo município no período $t$; $\operatorname{lnTrans} f_{i t}$ é 0 logaritmo das transferências correntes recebidas pelo i-ésimo município no período $t$; $\ln P I B_{i t}$ é o logaritmo do PIB; lnimp it é o logaritmo da arrecadação própria, enquanto $\varepsilon_{i t}$ é o termo de erro. A utilização das variáveis em logaritmo dos valores nominais segue o padrão da literatura.

Vale ressaltar que os gastos referem-se às despesas totais, ou seja, representa as cifras, em reais, alocadas para a realização das despesas municipais correntes - folha de pagamento, juros e encargos das dívidas, outras despesas.

A receita é aferida a partir da arrecadação dos impostos que correspondem à arrecadação própria do município, basicamente formada pelo Imposto Predial e Territorial Urbano (IPTU), Imposto sobre a Transmissão de Bens Imóveis Intervivos (ITBI-IV) e o Imposto sobre Vendas de Combustiveis e Lubrificantes (IVCL), bem como taxas e contribuições.

Por outro lado, as transferências expressam todos os repasses intergovernamentais correntes realizados pela União e Estado do Ceará aos municípios cearenses. Essas três variáveis foram extraídas do FINBRA, disponibilizada pela Secretaria do Tesouro Nacional.

O PIB é utilizado como proxy para a renda, além de ser uma medida para computar a atividade econômica do município, sendo mensurado em reais de 2000. E, por fim, a população considera os residentes em cada município. Essas duas variáveis são aferidas e disponibilizadas pelo IBGE.

O Quadro 1 reporta as variáveis, suas descrições, fontes e efeitos esperados sobre 0 gasto público. Vale ressaltar que as definições das séries expostas no quadro são condizentes com sua respectiva fonte.

Descritas as variáveis, se faz necessário descrever como são formadas as expectavivas (efeitos esperados) dos determinantes do gasto público. As relações previstas para a função gasto também reportadas no Quadro 1 podem ser justificados por questões ligadas à demanda (infraestrutura, saúde, educação etc.) e pela formação da receita municipal. Note que, todos os efeitos esperados são positivos, justamente pelo fato de variações na demanda e nas receitas proporcionarem mudanças nos gastos na mesma direção.

Variações no contingente populacional e/ou na atividade econômica provocam flutuações na demanda por serviços. Por um lado, um aumento do número de residentes em 
um dado município requer uma variação na oferta de serviços de saúde, educação, saneamento, urbanismo, etc., tal que se faz necessária uma elevação dos gastos praticados pelas prefeituras para atender às necessidades locais.

Aumento da produção, seja pelo setor de serviços, agropecuária ou indústria, requer um conjunto de investimentos capazes de garantir alguns dos fatores essenciais à instalação e manutenção da atividade produtiva, quais sejam: infraestrutura, qualificação da mão de obra, centros de coleta de resíduos industriais, serviços ambulatoriais etc. Portanto, espera-se que 0 crescimento econômico tenda a elevar os gastos dos municípios.

Como as receitas municipais são formadas por repasses do Estado e da União, bem como do seu esforço sobre a sua própria base tributária, é plausível supor que para um maior volume de recursos os governos locais sejam capazes de providenciar uma maior oferta de serviços. Portanto, acredita-se que uma variação nas receitas promova uma variação nos gastos

\section{Quadro 1 - Variáveis, Descrição, Fonte e Sinais esperados sobre o Gasto Público}

\begin{tabular}{|c|c|c|c|}
\hline Variáveis $^{* *}$ & Descrição & Fonte & Efeito Esperado \\
\hline Gasto & Despesa Corrente & FINBRA & ${ }^{* *}$ \\
\hline Receita & Arrecadação municipal & FINBRA & + \\
\hline População & Número de residentes & IBGE & + \\
\hline Renda & Produto Interno Bruto & IBGE & + \\
\hline Transferências & Transferências correntes & FINBRA & + \\
\hline
\end{tabular}

Fonte: Elaboração própria.

Nota: * variável dependente. ${ }^{* *}$ para estimar o modelo são consideradas as variáveis em logaritmo.

\section{ANÁLISE E DISCUSSÃO DOS RESULTADOS}

\subsection{Análise Descritiva}

Para subsidiar a análise do comportamento dos gastos públicos dos municípios cearenses, bem como dos seus respectivos componetes, a Tabela 1 apresenta um conjunto de estatísticas descritivas, para fundamentar um diagnóstico do perfil da gestão municipal cearense.

As estatísticas expressas na Tabela 1 apresentam algumas informações relevantes para análise do efeito flypaper. Inicialmente, pode-se destacar que a média das despesas municipais são inferiores à média dos repasses correntes realizados pelas esferas superiores de governo aos municípios. Contudo, tal fato é acompanhado por uma alta volatilidade, possuindo uma dispersão relativa à média superior a $430 \%$ e $380 \%$, respectivamente.
Portanto, apesar das observações dos valores médios indicarem uma postura de realizações de despesas de forma equilibrada, ou seja, sem elevações no déficit municipal, à dispersão absoluta apresentada pelos desvios padrão e pela variância, bem como a dispersão relativa retratada pelo coeficiente de variação, deixam em aberto qualquer conclusão sobre padrões imprudentes que preferem trabalhar a partir de recursos de terceiros a realizarem suas ações a partir de recursos próprios.

Verifica-se uma média populacional superior a 40 mil habitantes, o que indicaria uma boa quantidade de mão de obra disponível para a atividade produtiva, além de uma base tributária significativa. Contudo, os dados referentes a estas últimas variáveis evidenciam a fragilidade das fontes de arrecadação própria que não chegam a representar, em média, nem $1 \%$ dps valores observados para a despesa. 
Tabela 1 - Estatísticas Descritivas do comportamento fiscal dos municípios cearenses entre 1999 e 2009

\begin{tabular}{l|ccccc}
\hline & \multicolumn{5}{|c}{ Variáveis (em mil reais) } \\
\hline Estatística & Gasto & Receita & População & Renda & Transferência \\
\hline Média & $16.824,02$ & 25,47 & $42.872,39$ & 212,24 & $23.461,21$ \\
Desvio Padrão & $72.607,69$ & $219.092,70$ & 171.553 & $1.497,48$ & $89.798,74$ \\
Variância & $5,27 \mathrm{e}+09$ & $48.001,60$ & $29,4 \mathrm{e}+10$ & 2.242 .46 & $8.06 \mathrm{e}+09$ \\
Coef. de Variação & 4,31 & 8,60 & 4,00 & 7,06 & 3,8275 \\
Assimetria & 14,36 & 14,93 & 12,72 & 15,30 & 15,6989 \\
Curtose & 230,74 & 246,42 & 169,56 & 259,63 & 288,11 \\
Mínimo & 896,95 & 0,08 & 4.070 & 5,69 & 4,02 \\
Máximo & 1.418 .803 & $4.415,84$ & 2.505 .552 & $31.789,19$ & 1.999 .687 \\
\hline
\end{tabular}

Fonte: Elaboração própria.

Novamente, vale destacar a dispersão significativa apresentada pelos municípios cearenses em suas demais informações socioeconômicas - PIB, população e arrecadação - que apesar de possuírem uma dispersão mais moderada em relação às variáveis gasto e transferência, também se caracterizaram de forma elevada.

De acordo com os coeficientes de assimetria e curtose, pode-se caracterizar a distribuições das variáveis presentes neste trabalho como assimétricas a direita e leptocúrtica, respectivamente. Assim, as distribuições apresentam-se mais à esquerda do plano e com caldas mais pesadas. Tal fato reflete um perfil municipal concentrado em dois grandes blocos em torno da média, sendo aqueles à esquerda os mais presentes na amostra.

Apresentados os resultados das estatísticas descritivas sobre as características socioeconômicas dos municípios cearenses, a próxima seção dedica-se ao relato dos resultados obtidos a partir do modelo de dados em painel.

\subsection{Análise Econométrica, Estatística e Econômica do Modelo}

Inicialmente, foi definida qual estrutura melhor se enquadraria para análise do efeito flypaper. A escolha entre o modelo com efeito fixo e 0 de efeito aleatório foi balizada pelo teste de especificação de Hausman. A Tabela 2 apresenta o resultado para 0 referido teste, bem como os coeficientes estimados (e suas estatísticas).

Como se pode observar, o $p$-valor da estatística de Qui-quadrado é superior ao nível $5 \%$. Portanto, existem diferenças significativas nas estimativas do modelo com efeito fixo em relação ao modelo com efeito aleatório. Assim, a partir do teste de Hausman, optou-se pela utilização do modelo com efeito aleatório para se testar a hipótese do efeito flypaper junto aos municípios cearenses.

Primeiramente, são discutidos os resultados estatísticos do modelo e, em seguida, são realizadas as análises econômicas. Para análise estatística admite-se que os parâmetros estimados são estatisticamente diferentes de zero ao nível de significância de $5 \%$, pois $0 p$-valor para todas as estatísticas de teste individual são menores ou iguais a 0.05 . Assim, pode-se afirmar que 0 contingente populacional, a arrecadação, o PIB e as transferências afetam os gastos públicos dos municípios cearenses. Portanto, rejeita-se $\mathrm{H}_{0} \mathrm{e}$, admite-se que as estimativas são uma boa medida de sensibilidade para formação dos gastos.

Em relação às interpretações dos efeitos, verifica-se que as variáveis além de apresentarem um efeito individual, também possuem um impacto conjunto sobre 0 comportamento do gasto público municipal. Tal 
Tabela 2 - Resultado do modelo estimado com Efeitos Aleatórios, erro padrão robusto

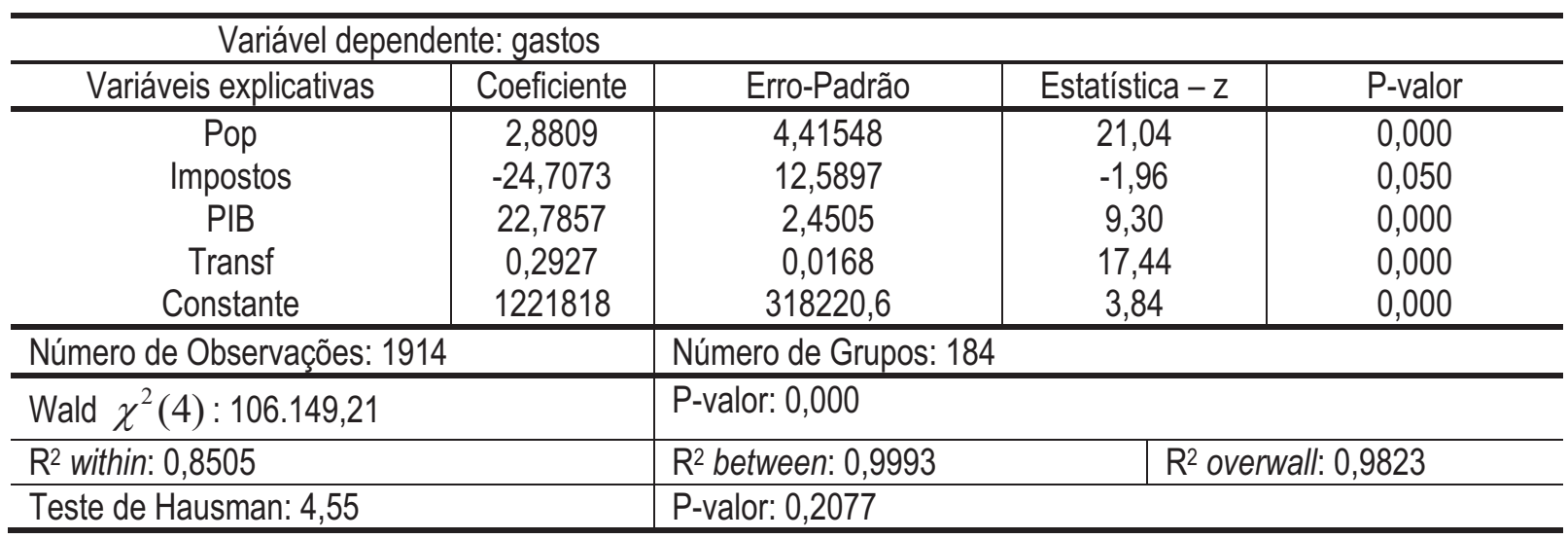

Fonte: Elaboração própria.

resultado é justificado pelo p-valor da estatística de Wald, que indica um efeito conjunto significativo ao nível de significância de $5 \%$.

Por fim, cabe destacar o bom ajuste para o modelo a partir dos resultados Whitin, Between e overall para o coeficiente de determinação, $R^{2}$, os quais apresentaram valores superiores a $85 \%$.

Feitas as argumentações dos resultados estatísticos, que forneceram os atributos necessários ao processo de inferência, passase à realização da análise econômica dos gastos municipais para aferir sobre a presença do efeito flypaper.

Em relação aos sentidos dos parâmetros, identificou-se que somente 0 coeficiente estimado para a arrecadação mostrou-se de forma divergente às expectativas formadas. Inicialmente, esperavase que a arrecadação, fonte de receitas próprias, seria responsável por afetar positivamente o gasto do município, já que 0 orçamento é formado de forma proporcional à receita tributária obtida no exercício anterior. Contudo, na prática, nos municípios não se observa esforço fiscal e, por isso, a arrecadação possui uma baixa representatividade na formação de receitas municipais. Sendo assim, é razoável supor (mesmo significante) que a arrecadação não determina a trajetória e as flutuações dos gastos públicos.

Na relação entre gastos e população, verifica-se que um crescimento do contingente populacional promove uma elevação nos gastos. Isto se faz necessário para a realização de novas despesas para satisfazer 0 equilíbrio entre oferta e demanda de serviços públicos. Tal fato é justificado pela estimativa do parâmetro referente à variável população, que indica uma elevação dos gastos governamentais de 92,88 reais, em média, para cada residente nos municípios cearenses.

O impacto da renda, representada pelo $\mathrm{PIB}$, mostrou-se de forma positiva sobre a realização de despesas. Sendo ambas as variáveis medidas em reais de 2000 , pode-se inferir que para uma elevação de 1 (um) real na produção, observa-se uma elevação nos gastos superior a vinte reais, dado que 0 efeito marginal da renda foi significativo e igual à 22,78 .

0 aumento da atividade produtiva oferece duas fontes de influências sobre os gastos públicos. Por um lado, o crescimento eleva a demanda por infraestrutura, além de agir como uma força de atração de novas atividades. Do outro lado, o mercado de trabalho reage positivamente ao crescimento da produção, promovendo um aumento da renda do trabalho e, consequentemente, do consumo de bens e serviços. Portanto, para atender as demandas da população, faz-se necessário uma ampliação dos gastos municipais.

Com uma estimativa significativa e com um sinal positivo, identificou-se uma relação positiva entre as transferências correntes e comportamento dos gastos realizados pelas administrações públicas municipais do Ceará. Como previsto, uma elevação nos repasses 
intergovernamentais geram um aumento das receitas nas esferas inferiores do governo, proporcionando uma maior disponibilidade à realização de novos gastos. Embora tenha apresentado um efeito marginal muito pequeno, observa-se que para cada real transferido temse uma elevação dos gastos inferior a 30 (trinta) centavos de real.

Por fim, faz-se uma comparação do efeito renda vis-à-vis com 0 da transferência, de forma que o saldo destes referidos efeitos proporcione 0 argumento necessário para satisfazer à indagação inicial, qual seja, se existe (ou não) efeito flypaper nas administrações públicas dos municípios cearenses. De acordo com as estimativas as elasticidades renda e transferência sobre os gastos públicos municipais são significantes e positivos, sendo que o impacto observado para a primeira é superior ao da segunda em mais de 75 vezes, indicando que a realidade pública municipal não condiz com a definição do efeito flypaper.

\section{CONCLUSÃO}

$\mathrm{Na}$ tentativa de avaliar o comportamento das administrações públicas municipais do estado do Ceará, no que diz respeito a sua gestão junto aos recursos, utiliza-se de uma função gasto para as referidas municipalidades de modo a oferecer uma estrutura capaz de fundamentar a análise do efeito flypaper.

Para realização de tal tarefa foram coletadas informações sobre as despesas totais, arrecadação própria, população, PIB e transferências correntes para os 184 municípios cearenses entre 1999 e 2009.

A divisão de recursos via transferências intergovernamentais são cifras que visam prover as administrações locais de recursos que os mesmos não dispõem e/ou não têm como dispor por não trabalharem de forma eficiente sua base tributária. Portanto, a importância da análise dos componentes das despesas públicas deve-se à possibilidade de exploração de recursos de forma inadequada para formação de receitas. Como a grande parcela das despesas é financiada por transferências, pelo menos em nível municipal, e sendo esta última uma fonte que não requer nenhuma atribuição para a referida esfera do governo, admite-se que os municípios tenham incentivos a priorizar suas atenções, como a obtenção de tais recursos em detrimento de sua base individual. É neste cenário no qual a análise do efeito flypaper está inserida.

A partir da estimação do modelo de dados em painel com efeito aleatório, pode-se dizer que as estimativas não identificam efeito flypaper por meio da análise da diferença dos efeitos da transferência e do PIB sobre os gastos públicos. Como o efeito marginal da renda, representada na função gasto pelo PIB, supera o notado para a transferência corrente, pode-se concluir que a prática descrita pela literatura como efeito flypaper não condiz com a realidade das administrações municipais do Ceará. Assim, mesmo podendo (ou não) trabalhar de forma eficiente suas receitas, os governos locais realizam suas receitas de forma mais efetiva para movimentos na renda do que para flutuações nos repasses intergovernamentais.

Por fim, cabe destacar o impacto significativo que a base tributária, expressa pelo contingente populacional e pela produção, tem sobre as despesas públicas. Como esperado, tais variáveis condizem com a demanda por bens/serviços e, portanto, induzem à geração de novos gastos.

Este estudo possui algumas limitações, entre as quais se dá destaque à não observância de informações mais longas para séries, o que impede a utilização de outras técnicas. Este trabalho utiliza-se de um modelo em painel que considera os parâmetros fixos no tempo, o qual poderia esconder ou não relatar a prática do flypaper em alguns momentos da amostra. A partir de uma maior dimensão temporal e também pela estrutura do modelo estado espaço, os resultados poderiam produzir parâmetros que variam no tempo de forma que fosse possível comparar os efeitos renda e transferências em distintos pontos e não somente pelo resultado médio. Contudo, tal fato não desqualifica os resultados obtidos, já que o resultado médio é formado a partir dos diversos pontos da distribuição e, portanto, funciona como uma boa medida de representação da população. 


\section{REFERÊNCIAS}

BRADFORD, D. F.; OATES, W. E. The analysis of revenue sharing in a new approach to collective fiscal decisions. Quarterly Journal of Economics, Oxford, v. 85, p. 416-439, 1971.

COSSIO, F.; CARVALHO, L. Os efeitos expansivos das transferências intergovernamentais e transbordamentos espaciais das despesas públicas: evidências para os municípios brasileiros. Pesquisa e Planejamento Econômico, Brasília, DF, v. 31, n. 1, p. 31, 2001.

COURANT, P.; GRAMLICH, E.; RUBINFIELD, $D$. The stimulative effects of intergovernmental aid: or why money sticks where it hits. In: MIESZKOUSKI, Peter; OAKLAND William $\mathrm{H}$. (Eds.). Fiscal federalism and grants-in-aid. Washington, DC: Urban Institute, 1979.

FILIMON, R.; ROMER, T.; ROSENTHAL, H. Asymmetric information and agenda control. Journal of Public Economics, [S. I.], v. 17, n. 1, p. 51-70, 1982.

GRAMLICH, E. M. Intergovernmental grants: a review of the empirical literature. In: OATES, $W$. (Ed.). The political economy of fiscal federalism. Lexington, MA: Heath-Lexington 1977.

; GALPER, H. State and local fiscal behavior and federal grant policy. Brookings Papers on Economic Activity, Washington, DC, 1, p. 15-25, 1973.

HAMILTON, J. The flypaper effect and the deadweight loss from taxation. Journal of Urban Economics, [S. I.], v. 19, p. 148-155, 1986.

KING, D. Fiscal tiers: the economics of multilevel government. London: George, Allen \& Unwin, 1984.

MATTOS, E.; ROCHA, F.; ARVATE, P. Flypaper effect revisited: evidence for tax collection efficiency in Brazilian municipalities. Estudos Econômicos, São Paulo, v. 41, n. 2, p. 239-267, 2011.

MCGUIRE, M. C. Notes on Grants- in-aid and Economic Interactions among Governments. Canadian Journal of Economics, St. Catharines, ON, v. 6, p.207-221, 1973.

OATES, W. E. Lump-sum intergovernmental grants have price effects. In: MIESZKOUSKI, Peter; OAKLAND William $H$. (Eds.). Fiscal federalism and grants-in-aid. Washington, DC: Urban Institute, 1979. p. 23-29.

On the measurement of congestion in the provision of local public goods. Journal of Urban Economics, [S. I.], v. 24, n. 1, p. 85-94, 1988.

QUIGLEY, J. M.; SMOLENSKY, E. Conflicts among levels of government in a federal System. Public Finance, London, v. 47, p. 202215, 1992.

SCHWALLIE, D. P. The Impact of Intergovernmental Grants on the Aggregate Public Sector. New York: Quarum Books Greenwood Press, 1986.

STRUMPF, K. A Predictive Index for the Flypaper Effect. Journal of Public Economics, [S. I.], v. 69, p. 389-412, 1998.

TURNBULL, G. Fiscal illusion, uncertainty, and the flypaper effect. Journal of Public Economics, [S. I.], v. 48, p. 207-223, 1992.

$\mathrm{NIHO}, \mathrm{Y}$. The optimal property tax with mobile nonresidential capital. Journal of Public Economics, [S. I.], v. 29, p. 223-239, 1986.

\footnotetext{
José Geraldo Araújo Correia

Pedagogo

Mestre em Economia pela Universidade Federal do Ceará (UFC)

Técnico de Controle Externo do Tribunal de Contas dos Municípios do Estado do Ceará (TCM-CE)

E-mail: geraldo@tcm.ce.gov.br
} 


\section{Guilherme Irffi Diniz}

Economista

Doutor em Economia

Professor adjunto da Universidade Federal do Ceará

(UFC)

E-mail: guidirffi@gmail.com

\section{Rodolfo Ferreira Ribeiro da Costa}

Economista

Doutor em Economia pela Universidade Federal do Ceará (UFC)

Professor adjunto da Universidade Estadual do Rio Grande do Norte (UERN)

E-mail: rodolfofrc@yahoo.com.br

Tribunal de Contas dos Municípios do Estado do Ceará - TCM-CE

Av. General Afonso Albuquerque Lima, 130 - Cambeba Fortaleza/CE

CEP: $60822-325$

\section{Universidade Federal do Ceará - UFC}

Av. da Universidade, 2853 - Benfica, Fortaleza/CE

CEP: $60020-181$

\section{Universidade Estadual do Rio Grande do Norte -} UERN

Rua Almino Afonso, 478 - Centro - Mossoró/RN CEP: 59.610-210 